\title{
Deciding the different robot roles for patient cognitive training
}

\author{
Antonio Andriellaa, ${ }^{\mathrm{a}, *}$ Guillem Alenyàa ${ }^{\mathrm{a}}$, Joan Hernández-Farigola ${ }^{\mathrm{b}}$, Carme \\ Torras $^{\mathrm{a}}$ \\ ${ }^{a}$ Institut de Robòtica i Informàtica Industrial CSIC-UPC \\ C/ Llorens i Artigas 4-6, 08028 Barcelona, Spain. \\ ${ }^{b}$ Fundació ACE. Alzheimer Treatment $\mathcal{E}$ Research Center \\ C/Marquès de Sentmenat, 57, 08029 Barcelona, Spain.
}

\begin{abstract}
Alzheimer's Disease (AD) and Mild Cognitive Impairment (MCI) represent a major challenge for health systems within the aging population. New and better instruments will be crucial to assess the disease severity and progression, as well as to improve its treatment, stimulation, and rehabilitation. With the purpose of detecting, assessing and quantifying cognitive impairments like $\mathrm{MCI}$ or $\mathrm{AD}$, several methods are employed by clinical experts. Syndrom Kurztest neuropsychological battery (SKT) is a simple and short test to measure cognitive decline as it assesses memory, attention, and related cognitive functions, taking into account the speed of information processing.

In this paper, we present a decision system to embed in robot that can set up a productive interaction with a patient, and can be employed by the caregiver to motivate and support him while performing cognitive exercises as SKT. We propose two different interaction loops. First, the robot interacts with the caregiver in order to set up the mental and physical impairments of the patient and indicate a goal for the exercise. This is used to determine the desired robot behavior (human-centric or robot-centric, and preferred interaction modalities). Second, the robot interacts with the patient and adapts its actions to engage and assist him to complete the exercise. Two batches of experiments were conducted, and the results indicated that the robot can take profit of the initial interaction with the caregiver to provide
\end{abstract}

\footnotetext{
${ }^{*}$ Corresponding author

Email address: aandiella@iri.upc.edu (Antonio Andriella)
} 
a quicker personalization, and also it can adapt to different user responses and provide support and assistance at different levels of interaction.

Keywords: Cognitive training, Assistive robot, Robot personalization, SKT, HRI

\section{Introduction}

The growing population of aging adults is increasing the demand for healthcare services worldwide. The number of people suffering from AD could cripple healthcare services in the coming decades [1], [2]. The latest estimation [3] says that 47 million of people suffering from dementia disease worldwide in 2016. This number will double by 2030 and will increase to more than 131 million by 2050. The number of older adults who have difficulties performing basic self-care tasks, called activities of daily living(such as dressing, bathing or eating), or instrumental activities of daily living (such as housekeeping, managing money or meal preparation) increases significantly with the prevalence of cognitive impairment. This is especially true for the population over 70 years [4]. If a person is not capable of performing these activities, continuous assistance from others is necessary. Most of these people need physical, emotional and cognitive assistance. The current healthcare systems struggle to provide adequate coverage of diagnostic services, and care is too often fragmented, uncoordinated, and unresponsive to the needs of people living with dementia [3]. Dementia doesn't only affect individuals. It also affects and changes the lives of their carers and families. Dementia has also a huge economic impact. The total worldwide cost of dementia in 2015 was US $\$ 818$ billion and it will become a trillion dollar disease by 2018 [3].

In order to detect, evaluate and quantify cognitive impairment different neuropsychological tools are used by clinical professionals. The SKT is a short test (10-15 minutes) that evaluates cognitive deficits in memory and attention through nine subtests: naming objects, immediate recall, naming numerals, arranging blocks, replacing blocks, counting symbols, reversal naming, delayed recall and recognition memory [5], [6]. Each subtest is limited to a maximum performance time of 60 seconds. Test scoring and auditing are also accomplished within a short time and are easily learned. The SKT correlates with other psychometric tests, such as: Mini-Mental State Examination, Alzheimer's Disease Assessment Scale [6] and Clock Drawing 
Test [7]. Also, it has shown correlation with brain Magnetic Resonance Imaging (MRI) [8] and Electroencephalogram (EEG) [5]. The test is highly accepted both by patients, given the characteristics of the test: short, simple and play-like and by hospital clinicians and general practitioners because of its brevity and simplicity.

The aging of society, if from one side represents one of the most important achievements of the last century, on the other side, the associated increase in healthcare requirements, represent a critical resource challenge for the healthcare system. Aging of society may affect economic growth and many other issues. There is a significant lack of support personnel such as caregivers and therapists, with concomitant reductions in the availability of the quality of the service. In view of this, there is a need to raise awareness of global aging issues and improve the efficiency of the care, especially in assistive and occupational therapy.

Socially Assistive Robotics (SAR) is a new field of robotics that focuses on assisting people through social rather than only physical interaction. The robot can provide emotional, cognitive and social cues to encourage development, learning or therapy for a person. In that way therapists could provide more consistent, effective training and monitor multiple patients simultaneously.

A recent study on cognitive stimulation demonstrated that an Integrated Psychostimulation Program is an effective tool to maintain cognition, functionality, and psychiatric symptoms in patients with dementia due to AD [9]. Also, this type of intervention that could be used by caregivers [10], has the potential to help diminishing the caregiver burden [11.

Recent studies consider that cognitive training interventions and, in particular, the use of games in mild dementia therapies is very promising in prevention and rehabilitation of elderly people [12]. Wiemeyer et al. [13] studied employing Serious Games (SG) in prevention and rehabilitation of elderly people. Recently, some studies have started to investigate the effectiveness of SG on people with $\mathrm{AD}$ and MCI and related mental disorders. McCallum et al. [14] proposed a literature review of a series of experimental results conducted to summarize the use of SG in neurodegenerative disorders. The results of the reported studies suggest that cognitive games can improve a number of cognitive functions, such as attention and memory/visual-spatial abilities. Unfortunately, some experts consider that SG are not well adapted to be played with a robot [?].

We believe that our paper is a step toward changing this perception, and 


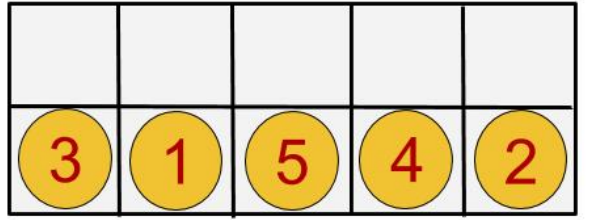

(a) SKT board initial setup

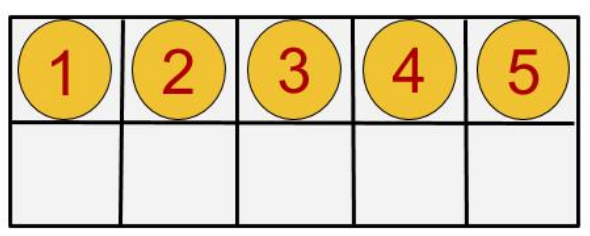

(b) board after completed SKT

Figure 1: Figure 1a shows the initial setting of the board (blocks are indicated in yellow), before the patient starts to perform the SKT. Figure 1b shows the board status after the patient complete the game with the support and assistance of the robot.

will present foundations to build a cognitive training robot able to support and assist a patient playing a modified version based on the SKT-subtest $V$ (replacing blocks). In the proposed game, numbered blocks are arranged randomly on the board, and at the patient is asked to replace the blocks in their corresponding numbered location. The objective is to complete the task as quickly as possible with as fewer mistakes as possible. Figure 1 shows an example of the board status before and after the test is completed.

Human emotions are difficult to represent and analyze [? ] but, when available, they can be used to provide better Human-Robot Interaction (HRI) experiences. In its current state, the proposed decision-making system does not account for such emotions, but the state representation can be easily extended with new literals to account for them.

We propose a robot/decision system with different levels of engagement to support:

- several levels of interaction;

- double loop HRI: Caregiver-Robot Interaction (CRI) and Patient-Robot Interaction (PRI);

- Human-centric vs Robot-centric.

We design the proposed system with 4 different use-cases in mind, defined as the next Personas [15]:

Bens : Ben 1 and Ben 2 are two patients with very mild dementia, Ben 1 has full mobility while Ben 2 has no mobility. The caregiver believes that the most appropriate strategy for Ben 1 is a human-centric one and configures the robot to mostly provide verbal encouragement, and 
to use physical support as a last resort. For Ben 2 the caregiver seeks to empower him, so he configures the robot to use the robot-centric strategy, where the robot asks the patient for help on the next move and physically executes the actions.

Bill : Bill is a patient with mild dementia and full mobility. The caregiver chooses the human-centric strategy, but configures the robot to provide verbal instructions (encouragement and support) preferentially.

Bob : Bob is a patient with severe dementia and full mobility. The caregiver chooses the human-centric strategy, but configures the robot to provide physical support preferentially.

In this paper, we investigate the symbolic interaction system, and evaluate it in simulation and in a simple scenario. Our approach focuses on the idea that SAR technology is able to provide affordable personalized cognitive assistance, encouragement, and companionship to individuals suffering from cognitive impairments related to aging, MDI or AD [? ].

As presented before, this work aims to validate that a robot/decision system can set up a productive interaction with a patient, and can be employed to motivate and encourage the patient about specific tasks or cognitive exercises like SKT. We do not tackle the problem of real speech recognition and voice synthesis, but assume that we can use one of the off-the-shelf libraries available from Google, IBM, or Microsoft. At the moment, we want to validate the planning approach and the experiments are still not performed with real users.

\section{Related work}

The literature in assistive robotics that tries to address the aforementioned problem is quite limited. Montemerlo et al. [16] designed a robot able to guide elderly individuals in an assisted living facility, a time-consuming task currently carried out by nurses and alerting and informing them of an upcoming event or appointment. Kidd et al. [17] used a seal robot, named Paro, in their experiment protocol and they observed it has features that can increase social interactions. This effect is enhanced in the presence of caregivers willing to participate in the interactions. The kind of interactions provided is not only pleasing, feel-good experiences, but also evocative experiences for the users. Another work in this direction was published by 
Matsusaka et al. [18]. They developed a humanoid that can demonstrate physical exercises in collaboration with the human demonstrator. Through the experiment, they confirmed that the robot is effective in stimulating the user for health exercises. Hamada et al. [19] proposed a new recreation program using a robotic dog, named AIBO, with a new evaluation method for caregivers. The program consists of two games, designed to induce people to perform specific actions. The preliminary results show an improvement of people ability not only proved by the evaluation of the caregiver but also on the proposed evaluation criteria. McColl et al. 20] investigated user engagement and accordance during meal-time interactions with the robot. They demonstrated that participants enjoyed interaction with it and had a positive attitude toward the robot for that kind of activity. Tapus et al. [21] presented a SAR technology that aims to provide a customized support and assistance to users suffering from cognitive impairment.

Previous studies investigating the role of embodiment in the human-agent context have demonstrated the potential positive effects that the use of real robots can have on people's level of engagement and the perception of the agent they are interacting with. Brainbridge et al. [22] explored how the physical presence of a robot influences human evaluations of the robot as a social partner in book-moving tasks with a humanoid robot that was physically present or displayed via a live video on a screen. Several tasks were created and then examined to evaluate the following aspects of social interactions: greetings, cooperation, trust, and personal space. Users were more opened and available to perform an unusual request and afford more personal space to the agent when they interact with a physically present robot with respect to interactions with a live video feed of the same robot on a computer screen. Power et al. [? ] compared interactions between robots and similar computer-agents that engaged participants in a conversation in a health interview. They found that participants spent more time with the collocated robot and their attitudes were most positive toward it. Wainer et al. [? ] studied the effect of a physical embodiment on the performance and impression of social interactions. The results of a set of experiments suggested fundamental differences between virtual agents and robots from a social standpoint and it would also have significant implications for HRI.

Current research in assistive robotics don't elaborate on certain aspects that we believe are considerably important, especially in a cognitive game scenario like the one we will present in this paper.

First, the role of the caregiver has not been sufficiently considered. Most 
of the works tried to replace him with the robot, leaving the whole complexity of the interaction with the patient. On the contrary, we believe that the caregiver has to be in the loop of interaction for: i) providing a correct setup to the robot and ii) intervening in the situations that the robot cannot manage.

Second, there is not much work on using robots to administer cognitive games in general, none about SKT. This test is mostly used in healthcare centers to monitor attention and trace memory. It provides a powerful tool for improving the effectiveness of the caregivers and the acceptance by the patient. We believe that introducing it, first, for cognitive evaluation and in the future for cognitive training can have an high impact especially in the healthcare community.

As last aspect, the approach we propose based on the usage of planning for decision making has not been explored in this specific context.

\section{Proposed method}

\subsection{Levels of interaction (PASS)}

Robots can exhibit multiple interaction modalities with people, either patients or caregivers. We relate the different levels of interaction to the widely used PASS (Performance Assessment of Self-care Skills) [23] that defines 9 levels for self-care applications. PASS was deployed to determine the users' capacity for community living, and it suggests that occupational therapists must use measures that capture the person-task-environment transaction and compare users' task performance to a performance standard. In the paper the authors describe tasks that users from various diagnostic populations could and could not perform independently and safely. The assistance levels, from the least to the most assistive are:

1. verbal supportive (encouragement to initiate, continue, or complete a task)

2. verbal non-directive (cues to facilitate task initialization, continuance, or completion without telling the user exactly what to do)

3. verbal directive (statements to initiate, continue or complete a task)

4. gestures (tactical cues to inform the user on how to initiate, continue or complete a task, e.g. pointing at an object)

5. task/environment rearrangement (break task down) 
6. demonstration (demonstrate task/subtask combining with verbal statements to illustrate how to initiate, continue, or complete a task)

7. physical guidance ('hands down' - movement of the user's body as needed to facilitate an action to promote task initialization, continuation or completion body part needed)

8. physical support ('hands up' - contact with the user to support the body or an extremity to promote task initialization, continuation, or completion)

9. total assistance (do task/subtasks for the person by compensating for the person's disability as appropriate for the underlying impairments)

In the current work, we implement levels 1, 2, 3, 4, 6 and 9, mostly used into the PRI. Although the original PASS defines only gesture for level 4 , we decided for that level to combine gesture with one of the assistance levels defined in 1,2 or 3. The main reason is that in HRI a support with only gesture is not as effective as combining it with speech. As it will be shown in the next sections, this level is particularly worth for a robot-centric perspective.

\subsection{Planning for decision making}

In the proposed method we aim to use planning as the decision making framework. The two most common alternatives in this case are Finite State Machines (FSM) or a Behavior Trees (BT). In the case of FSM, the formalization of the domain (using if-then-else conditions) would have been easy to understand and implement for a simple case. However, FSM is known to have difficulties in complex scenarios and, in particular, the variability in the user reactions and the adaptability that is expected from the robot are difficult to encode. The resulting FSM would be complex and hard to maintain.

In the case of BT, there is a higher possibility to have more refined conditions and strategies. Tasks have a common structure (they are allocated CPU time to perform an action and return success or failure) and are divided into: i) leaf tasks, which check a condition or execute some code, and ii) composite tasks, which return a value depending on child tasks. They are commonly used in scenarios where there is no need to have a mathematical foundation incorporating continuous-time dynamics. However, such requirement has to be taken into account in order to use BTs on more complex applications, e.g. real robots, control systems [24]. 
Both FSM and BT are reactive techniques, they follow a pre-programmed strategy that specifies how the robot should react depending on the current state/node and the conditions that currently hold in the domain. A sequence of actions that can be executed in the SKT game need to be represented explicitly in the structure of the FSM or BT.

When the possible interactions could increase and the robot would need to be autonomous, with its own behavior and goals, maintaining the possible and applicable interactions using those techniques may become complex and difficult, and more flexible techniques are needed. A planner uses predicate logic to model the actions an agent can perform. Using this action model, the information about the current state contained in the inner world, and the task the system must perform, the planner can generate a sequence of high-level actions to be executed. The main advantages are: i) facility in managing a large number of generated behaviors, ii) ability to achieve different behaviors under different pre-conditions without explicitly listing all the resulting strategies.

The domain and actions are formalized using Planning Domain Definition Language (PDDL) [? ], which is an action-centered language inspired by Stanford Research Institute Problem Solver - like (STRIPS) formulations of planning problems. At its core, there is a simple standardization of the syntax for expressing this familiar semantics of actions, using pre- and postconditions to describe the applicability and effects of actions. An early design decision in the language was to separate the descriptions of parameterized actions characterizing the domain from the problem description. The same domain description can be paired with many different problem descriptions to yield different planning problems in the same domain. The pre- and postconditions of actions are expressed as logical propositions constructed from predicates, argument terms and logical connectives.

As a planning system, we decided to use Metric-Fast Forward (Metric-FF) [? ]. Metric-FF is a state-of-the-art planner and it is an extension of FastForward $(\mathrm{FF})$, which supports numerical plan metrics, that is an interesting feature to keep into account in an optimization process. Metric-FF accepts domain and problem specifications written in PDDL 2.1 level 2. Apart from the well-known Action Description Language (ADL) features, this allows for a finite number of numerical state variables. At any point in a (pre-, effect-, or goal-) condition formula where a logical atom is allowed, it is possible to set a numerical constraint, for a comparison between two expressions over the rational numbers and the numerical variables. Therefore optimization crite- 


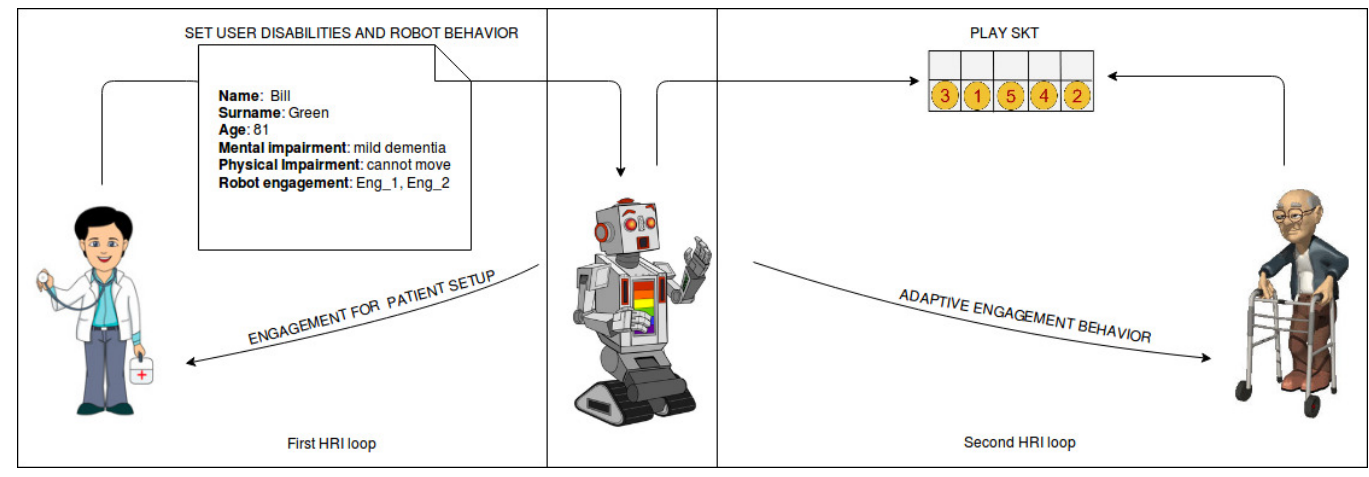

Figure 2: Double loop of interaction: Caregiver-Robot Interaction (CRI) and PatientRobot Interaction (PRI).

ria can be specified inside the problem file, e.g. with the statement(:metric minimize(cost)). The system deals with PDDL 2.1 level 2, combined with ADL. A PDDL definition consists of two parts: the domain and the problem definition. The domain definition contains the domain predicates and actions of the actors involved in the interaction. The problem definition contains the objects present in the problem instance, the initial state description and the goal to be achieved (see Appendix A).

\subsection{HRI Decision System in a SKT Scenario}

Our goal is to design a decision system able to provide assistance and support, mainly focused on assessing the cognitive abilities of a patient with dementia. Based on the PASS levels of interaction, we first interact with the caregiver to provide the patient a better engagement during task execution and increase the positive effect.

We aim to keep the patient constantly stimulated and engaged during the entire test using the personalized setting for their level of impairment.

A decision system based on a generic modular PDDL is proposed to allow the robot to provide: i) interaction with the caregiver to personalize the robot for a specific patient and ii) interaction with the patient to assist and support him as he engages in a cognitively stimulating test like SKT as shown in Figure 2.

The HRI system focuses on determining the patient's actions (move or no move) and his task performance during the interaction with the robot, according to the level of interaction and consequently of assistance, defined by the caregiver. 


\begin{tabular}{|l|l|}
\hline \multicolumn{1}{|c|}{ Caregiver setting } & \multicolumn{1}{c|}{ Robot interaction } \\
\hline Patient cannot speak & All the levels of engagement are allowed \\
\hline Patient cannot move & Only the demonstration level is allowed \\
\hline Patient with no impairments & All the levels of engagement are allowed \\
\hline
\end{tabular}

Table 1: Caregiver settings for patient's physical disabilities

\subsection{Modeling the caregiver preferences}

In Figure 2, we show the first loop of HRI involving the caregiver. In the CRI, the robot engages the caregiver before starting an SKT session with a patient. Although the interaction is very simple and at the moment only the mental and physical disabilities of a patient can be modeled, we consider it a milestone for two main reasons.

First, because one issue of HRI in rehabilitation and assistive contexts is the acceptance from the caregiver of the robot abilities [? ]. If the caregiver has confidence in the robot he will cooperate with it for the task completion. Secondly, it allows to personalize the robot behavior for that specific patient so as to provide him with the best setting to achieve the task.

The caregiver may support the robot also during task execution, changing the robot's level of interaction if it is not suitable for the current patient.

Table 1 illustrates the caregiver's possible choice among three different settings for physical disabilities: patient that cannot speak, patient that cannot move or patient with no impairments. According to the caregiver selection, the robot will exclude some levels of engagement and assistance. For instance, if the patient is not able to move his arms and consequently he can not perform the task to move a block by himself, the caregiver could ask the robot to perform the movement for him.

Then, the robot asks for the mental impairment level of the patient (Table 2). At the moment, we defined three levels: very mild dementia, mild dementia and severe dementia. Based on the medical history of the patient, the caregiver can also decide to personalize the engagement levels.

For example, the predefined engagement for a patient with severe dementia is expected to be Eng_3 and Eng_4 (Table 2), but the caregiver may decide to select Eng_2 instead of Eng_3.

It is worth highlighting the importance of the caregiver role in this phase: a mistake in choosing the levels of interaction may affect the scope of the test. Selecting a higher level of disability could result in loss of engagement 


\begin{tabular}{|c|l|}
\hline Caregiver setting & Robot interaction action \\
\hline \multirow{2}{*}{ Patient with very mild dementia } & Eng_1 - Encourage \\
\cline { 2 - 2 } & Eng_2 - Suggest subset \\
\hline \multirow{2}{*}{ Patient with mild dementia } & Eng_2 - Suggest subset \\
\cline { 2 - 2 } & Eng_3 - Suggest solution \\
\hline \multirow{2}{*}{ Patient with severe dementia } & Eng_3 - Suggest solution \\
\cline { 2 - 2 } & Eng_4 - Demonstration \\
\hline
\end{tabular}

Table 2: Predefined engagement level settings

with the patient because part of the task will be performed by the robot. On the contrary, selecting a lower level of disability could result in no proper assistance by the robot with a consequence of patient frustration by not having achieved the goal or discouragement by having spent too much time to achieve it.

\subsection{Adaptive Robot behavior in the SKT scenario}

The robot's behaviors for the SKT game activity seek the overall objective of assisting the patient during the test, identifying and checking the correct blocks on the grid according to the task selected by the caregiver. The behaviors are categorized into providing:

1. encouragement - according with level 1 of PASS test

2. suggestions (e.g. giving a subset of blocks) - level 2 of PASS test

3. statement to complete the task (e.g. suggesting the correct move) level 3 of PASS test

4. gestures to assist or complete the task (e.g. pointing out an area or the location of the correct move) - level 4 of PASS test

5. demonstration (e.g. grasp the block and move it in the correct position) - level 6 of PASS test

6. full assistive - level 9 of PASS test

In our scenario, as mentioned in the introduction, we do not deal with problems related to speech and gestures detection, we assume the robot can perceive the user's actions.

For each level of engagement presented in Table 2, we defined a corresponding robot action, that means the robot perceives a change in the state of the patient (move or no move) and decides to engage him, starting to support him in accordance with the caregiver setting. 


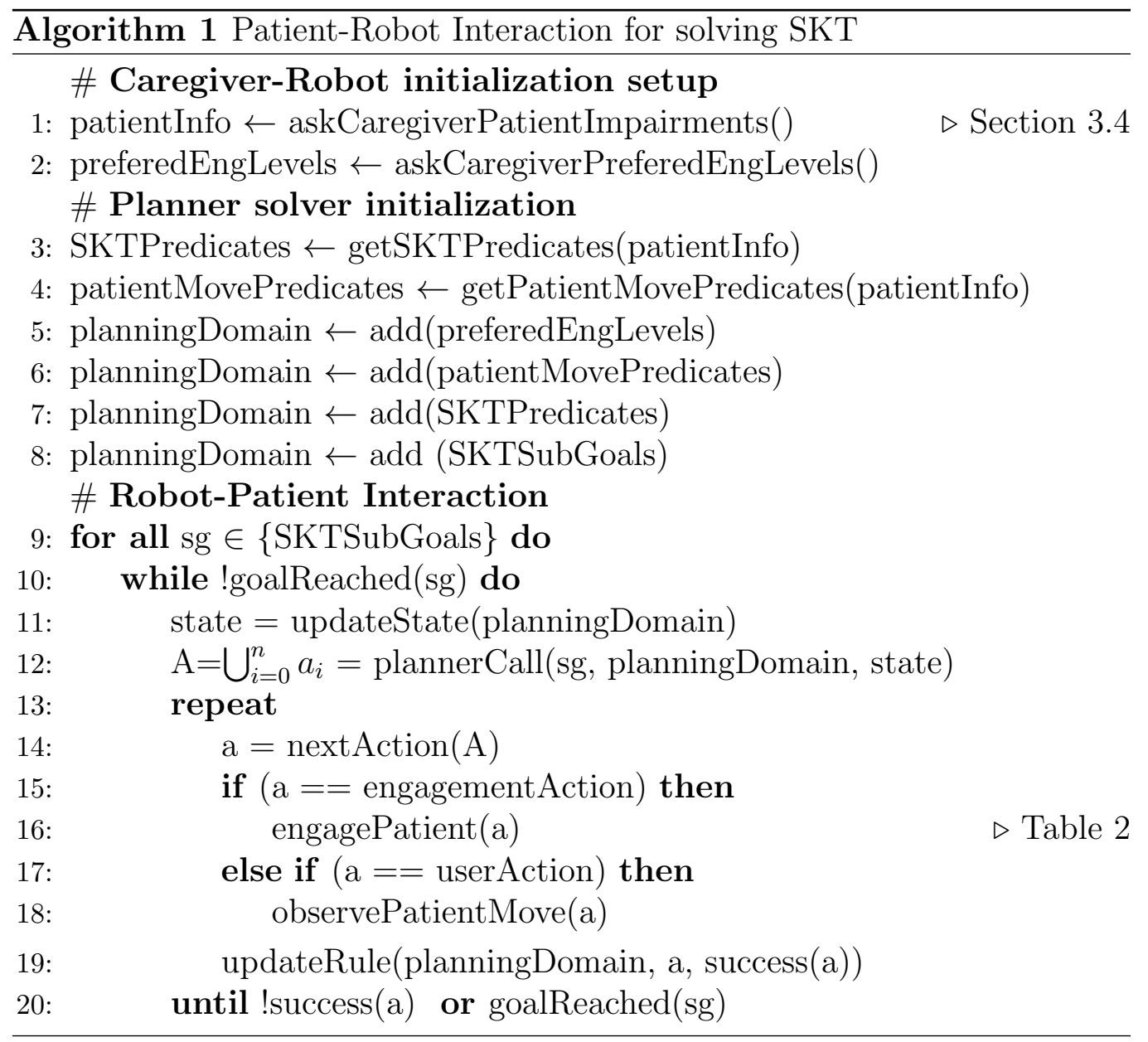

Algorithm 1 describes the PRI to complete the SKT. The Initialization step (lines 1-2) provides the output of the caregiver setting. If no CRI is provided, the robot uses the same interaction levels for all the patients (Eng_1,..,Eng_4). Then (lines 3-7), the robot creates and populates the domain definition with predicates and actions, and the problem definition with the initial state and the goal to achieve for the Planner Solver (Metric-FF).

It is important to notice that at line 3 the caregiver defines the SKT specification. For example, he defines the number of blocks and test goal. We define the goal $G$ as the state in which all the blocks are replaced in the correct location on the board. With the same logic we define the subgoal $s g$ as the state where one block is replaced in the correct position on the board. 
A related point to consider is that the task of our planner is to manage the interaction and not solving the game, and thus we assume that the goal and the intermediate subgoals are available. This is a reasonable assumption that can be manually encoded for simple games or solved using a game solver.

Lines 9-20 describe the main steps of the algorithm defining the interaction between the robot and the user.

In this phase the planner has a central role, because it is responsible, according to the current state, to define the sequence of the actions to perform in order to reach the goal.

Firstly, we define an outer loop (line 10) that iterates through each of the subgoals defined in the initial setup (line 8). Until a subGoal is not reached, we get the current state, and updating it with the predicates that are valid in the planningDomain (line 11). Then the system calls the FF-Metric planner, that takes as input, the current subGoal sg, the domain planningDomain, and the current state state (line 12) and returns the sequence of the actions $A$. For each of the action dispatched by the planner (lines 13-14) the system parses it to define which kind of action has to perform. Based on the kind of action, the robotic decision system elaborates it in a different way (lines 15-18).

It is important to notice, that what we define as userAction (line 17) and observePatientMove (line 18) are respectively the action dispatched from the planner and the observation of the robotic decision system about the user action. For the sake of simplicity, we haven't reported in Algorithm 1 that both the actions includes three different subactions (grasp, move, release) as described in the implementation in Appendix A.

Finally, the function updateRule (line 19) updates the predicates in the planningDomain. The decision system stops processing actions only when one of the two conditions are faced: the goal is achieved or the action failed (line 20). In the first case the robotic decision system moves to the next subGoal while in the other case, the planner based on the current state representation re-plans a new sequence of actions. An example of the predicates valid in our planning domain, when the subgoal is achieved is listed below.

- (at t1 11)

- $\operatorname{not}($ free_loc 11)

- (free_loc 18) 


\begin{tabular}{|c|c|c|}
\hline Approach & Robot interaction & Example of behavior \\
\hline $\begin{array}{l}\text { human-centric } \\
\text { robot-centric }\end{array}$ & Instruction & $\begin{array}{l}\text { Hi, I'm SOCRATES. I will play the test with you. } \\
\text { The goal is to replace all the blocks in the correct location. } \\
\text { Please try to be as fast as possible. Let's start! }\end{array}$ \\
\hline human-centric & Encouragement & $\begin{array}{l}\text { I know you can do it. } \\
\text { Let's move a block! }\end{array}$ \\
\hline human-centric & Suggest subset & $\begin{array}{l}\text { Unfortunately that move is not correct. } \\
\text { The correct one could be one among: Px, Py, Pz. } \\
\text { Please try again and keep in mind my advices! }\end{array}$ \\
\hline human-centric & Suggest solution & $\begin{array}{l}\text { Although you're very close to the solution, } \\
\text { that was not correct. } \\
\text { The correct position for Px is Lx. } \\
\text { Please try again, come on! }\end{array}$ \\
\hline robot-centric & Encouragement & $\begin{array}{l}\text { I don't know what to do. } \\
\text { Could you provide me an advice? }\end{array}$ \\
\hline robot-centric & Suggest subset & $\begin{array}{l}\text { Unfortunately your previous suggestion was not right. } \\
\text { I know that the solution is one of the following: } \\
\text { Lx, Ly, or Lz. } \\
\text { Could you try again? I know you can help me! }\end{array}$ \\
\hline robot-centric & Suggest solution & $\begin{array}{l}\text { Although I think we're close to the solution, } \\
\text { that was not right. } \\
\text { I know the correct position for Px is location Lx. } \\
\text { Could you tell me if I'm right? }\end{array}$ \\
\hline $\begin{array}{l}\text { human-centric } \\
\text { robot-centric }\end{array}$ & Demonstration & $\begin{array}{l}\text { The correct position for block Px is Lx. } \\
\text { Look at me, } \\
\text { I'll show you how to perform the move! }\end{array}$ \\
\hline $\begin{array}{l}\text { human-centric } \\
\text { robot-centric }\end{array}$ & Congratulation & $\begin{array}{l}\text { Congratulations, we have made a successful move. } \\
\text { We're not so far way to complete the test. } \\
\text { Keep on it! }\end{array}$ \\
\hline
\end{tabular}

Table 3: Example of robot engagement behaviors for the SKT

- (released bob t1 18 11)

In that state, Bob has moved the block $t 1$ from location $l 8$ in the correct location $l 1$, so the block is not anymore in its initial position $l 8$ and it has been released in location $l 1$.

As an example, we describe below two different scenarios in which the robot selects a Human-centric approach to interact with Ben 1 in the first case, while in the second a Robot-centric approach to interact with Ben 2 is chosen. A summary of the robot's behaviors related to each level of interaction is in Table 3 ,

In detail, First scenario:

1. Robot gives instruction to patient on how to perform the test. 
2. Robot engages patient for the first time (Eng_1) : "Come on Ben, make a move. I know you can do it".

3. Patient moves a block.

4. Robot identifies a wrong move.

5. Robot engages patient for the second time (Eng_2): "The first move was not the right one. The correct block is one of those: Px, Py and $\mathrm{Pz}$, try again and keep in mind my suggestion".

6. Patient moves a block.

7. Robot identifies a correct move from the patient and congratulates with him.

Second scenario:

1. Robot gives instruction to patient on how to perform the test.

2. Robot engages patient for the first time (Eng_1): "I don't know what to move, can you give me a suggestion?"

3. Patient gives a suggestion to the robot.

4. Robot identifies a wrong move.

5. Robot engages patient for the second time (Eng_2): "That one was not the correct move, I know it should be one among $\mathrm{Px}, \mathrm{Py}$ and $\mathrm{Pz}$, but I'm not 100\% sure. Could you provide me the solution? I know you can help me!"

6. Patient gives a solution to the robot.

7. Robot identifies a correct move from the patient and congratulate with him.

As mentioned in Section 3.3, we formalized our domain using a logic formalism. In Figure 3 we show, as an example, the formalization of a grab action in PDDL pseudo-code. That action can be performed by an agent: robot or patient. In the case of the robot, gripper is a robot arm, block is the block itself to be grabbed and from is the location where the block is. As pre-condition the robot needs to check if: the block is in that location; the final destination of the object is not already occupied by another block; the block is not already caught and then the robot arm is free. As effects, the robot now has caught the block and his arm is not free anymore.

For a better understanding, it is worth noting that the other actions formalized for an agent are: move and release. The former defines that the agent has already the block in his hand and, it is moving it toward a location of the board. The latter is the action of releasing a block in a defined location. 


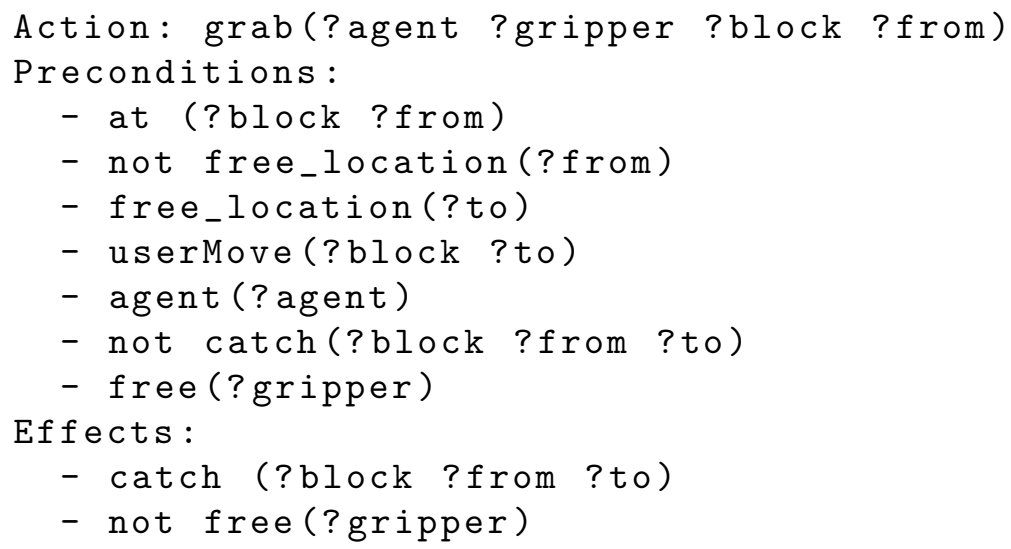

Figure 3: Example of grab action in PDDL pseudocode

According to Table 2, we show an example of robot's actions for gesture and demonstration engagement, respectively.

For instance, action robot_point_area_of_the_board, is the second level of interaction for gesture engagement. The robot points to an area of the grid and asks the patient to guess the correct move. The action performed by the robot is shown in Figure 4.

Lastly, action robot_demonstrate_move is the forth level of engagement. That level is usually defined by the caregiver for patient with severe dementia. The robot shows the patient how to perform the move in the correct way (Figure 5).
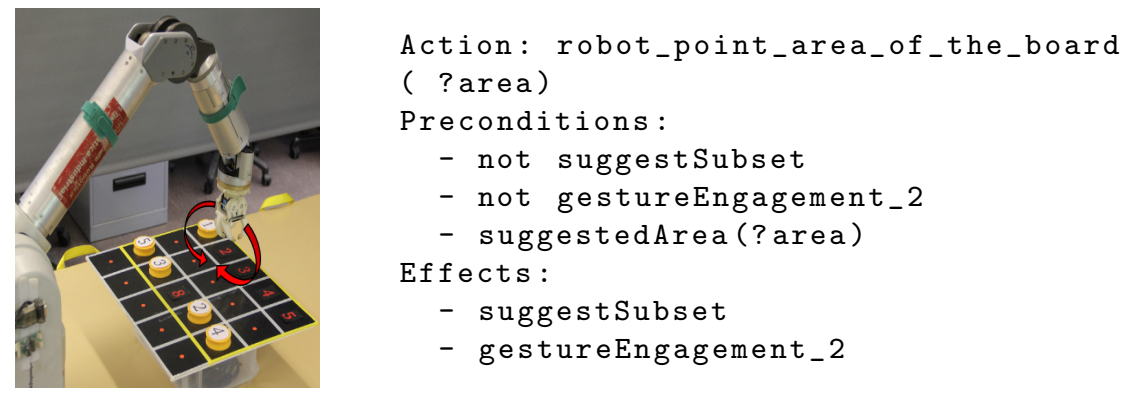

Figure 4: The robot suggests the user where to move a block by pointing to an area on the board.

The complete problem representation and domain description is described in Appendix A that includes a link to the implementation of PRI in PDDL. 

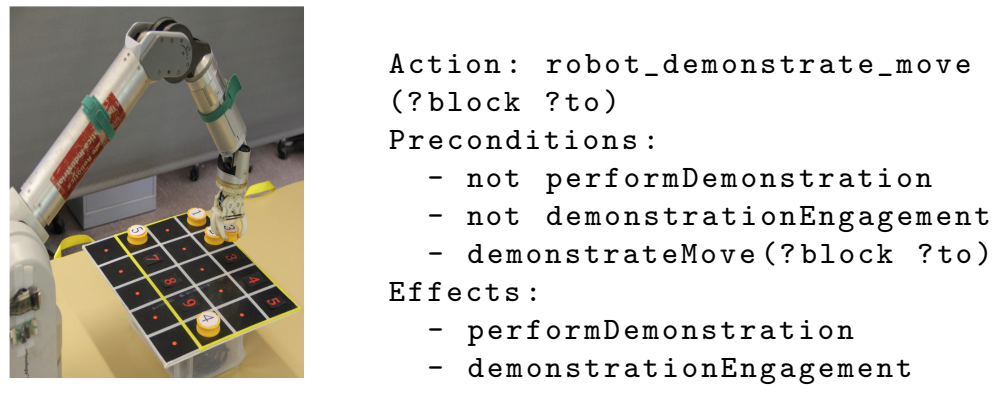

Figure 5: Robot shows the user how to perform a move

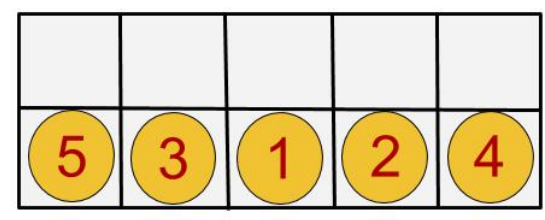

Figure 6: Initial setting scenario for experiments.

\section{Experiments}

In the following Section we provide some experiments to validate that a decision system can interact with a patient and support him in performing a subtest of the SKT. The behaviors of the robot, patient and caregiver are simulated.

In Section 4.1, the setup used in our simulated system is discussed. Section 4.2 and 4.3 show the results of playing SKT without and with a CRI, respectively. Finally in Section 4.4, we discuss and compare the results of the two simulations.

\subsection{Experiments setup}

The initial setup of our scenario is shown in Figure 6. Five numbered blocks (from 1 to 5 ), were placed randomly on a board (2x5). Each cell on the board was numbered from 1 to 10. At the beginning the blocks were located on the second row (from 6 to 10). The patient had to place each numbered block in its corresponding numbered cell on the board. The time to complete the game and the number of times the patient needs support (number of engagements) constitute the score recorded. 


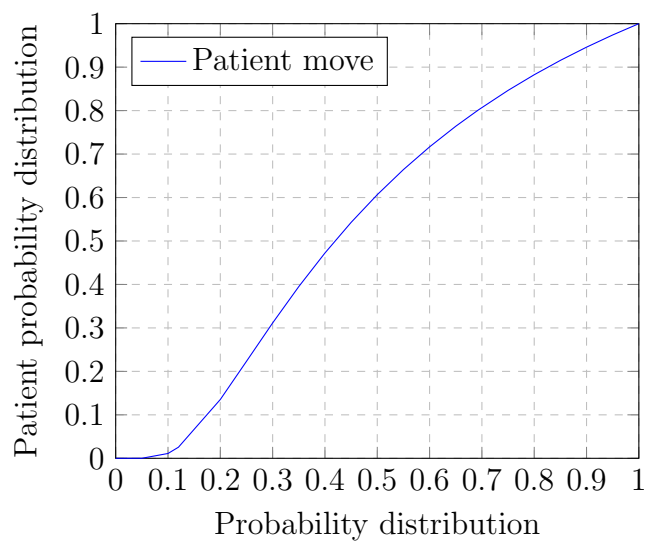

Figure 7: Exponential distribution of patient move

\begin{tabular}{|c|c|c|}
\hline Patient & Probability & Mental Disability \\
\hline Bob & 0.1 & severe dementia \\
\hline Bill & 0.5 & mild dementia \\
\hline Bens & 0.7 & very mild dementia \\
\hline
\end{tabular}

Table 4: Patients' probability to guess the correct move

All the experiments were performed in simulation. We conducted 10 tests for each 3 use-cases (Bens, Bill, Bob) and for each of those, we repeated the tests with two different settings. The first one without the caregiver interaction loop while in the second the caregiver was engaged. We decided to consider Ben1 and Ben2 as only one person, because with the current settings, there was no difference if the actions were performed from the user or from the robot. All the results shown in the next sections have been averaged over the 10 tests performed for each setting.

To simulate the patient's mental impairments, we modeled the probability of a patient to guess the correct block, as a three steps procedure. At first we simulated a value uniformly between 0 and 1 , then this value was mapped using the function in Figure 7. At the end, if for a given patient the resulting value was below the threshold in Table 4 , we considered a move as a right one.

Notice that if we did not apply the exponential transformation, or, equivalently, if the function was a straight line, the move was Bernoulli distributed, while using the exponential function, we introduced more flexibility. 


\begin{tabular}{|c|c|}
\hline Engagement level & Probability reward \\
\hline 1 & 0.05 \\
\hline 2 & 0.1 \\
\hline 3 & 0.3 \\
\hline
\end{tabular}

(a)

\begin{tabular}{|c|c|}
\hline Patient move & Time to complete action \\
\hline correct move & $1 \mathrm{~s}$ \\
\hline wrong move & $3 \mathrm{~s}$ \\
\hline doing nothing & $10 \mathrm{~s}$ \\
\hline
\end{tabular}

(b)

Table 5: Table 5a shows the probability rewards corresponding to each level of engagement. Table 5 b shows a symbolic time competition defined for each patient action.

There were two main reasons to introduce an exponential function to map a uniform distribution: first, having a non linear distribution of the patient's moves gave us the opportunity to model in a different way the three patients, and secondly, in this way we provided a more coherent mechanism of reward for each patient at each level of engagement (Section 3.4 Table 2).

Table 5 a summarizes the rewards corresponding to the different levels of engagement. The higher the engagement, the higher the reward. This system of rewarding allowed to emulate a possible behavior of a patient after a robot engaged him.

With that idea in mind, the exponential curve helped us also to model how the rewards had a different effect for each patient. It would appear reasonable that an encouragement or a suggestion to Bens, that had a very mild dementia, had not the same effect on Bob, that had a severe dementia, in terms of guessing the next move.

During the test the patient was assisted and supported by the robot. Each simulated patient could:

- grab, move and release a block in the correct position

- grab, move and release a block in the wrong position

- do nothing, that means he was not capable to decide what was the next move or he lost attention or focus on the task.

A simulated decision system (robot) could:

- interact with the caregiver to set the engagement levels of the patient

- interact with the user with the defined levels of engagement using speech and/or gesture

A simulated caregiver could: 


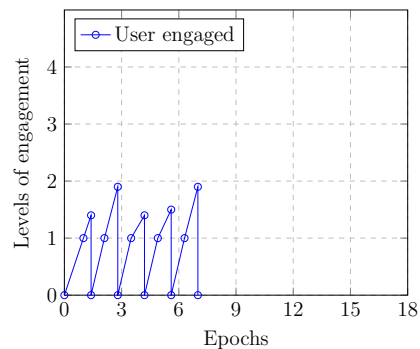

(a) Bens very mild dementia, average time $22 \mathrm{~s}$
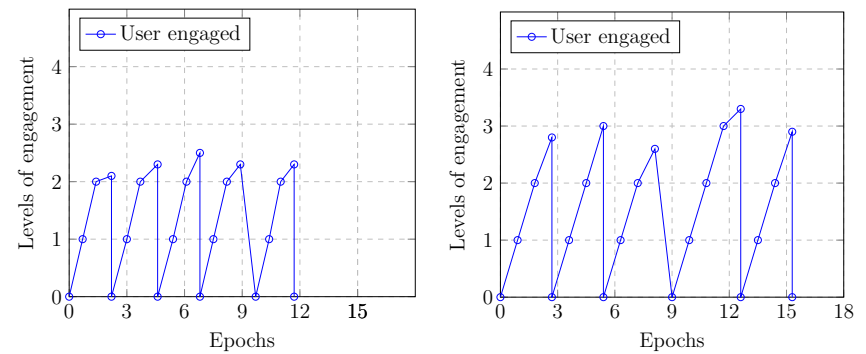

(b) Bill mild dementia, (c) Bob severe dementia, average time $47 \mathrm{~s}$ average time $75 \mathrm{~s}$

Figure 8: The plots show the interaction levels employed by the robot in order to support and assist the patients without a first CRI. Figures 8a, 8b, 8c show the evolution in time of Bens, Bill and Bob, respectively, playing SKT.

- interact with the robot to define the engagement levels for the patient

For each action of the patient, the robot will react based on its stateaction representation for that situation. Each time the patient will make a wrong move or do nothing action, the robot will try to engage him. We assigned a symbolic time to complete each of the three actions described above, as shown in Table $5 \mathrm{~b}$.

The goal was to complete the task in as less time and with less support (number of engagement trials) as possible. Outcomes were quantified by evaluating task performance and time on task.

\subsection{Experiments with no Caregiver-Robot Interaction}

In this first batch of experiments, the objective was to evaluate how a simulated user could be supported and assisted by a robot, which was not aware of the specific physical and mental impairments of the patient and, consequently, it interacted with all of them (Bens, Bill, Bob) with the same level of engagement.

Figure 8 shows the evolution over epochs (that in our case were attempts of moving) of the SKT subtest in terms of number of moves and levels of engagement triggered to achieve the goal.

In Figure 9a we summarized the number of engagements for each level of interaction.

Figure 8a shows the evolution in time of Bens playing SKT. As we expected, patient Bens was able to complete the game without requesting too 


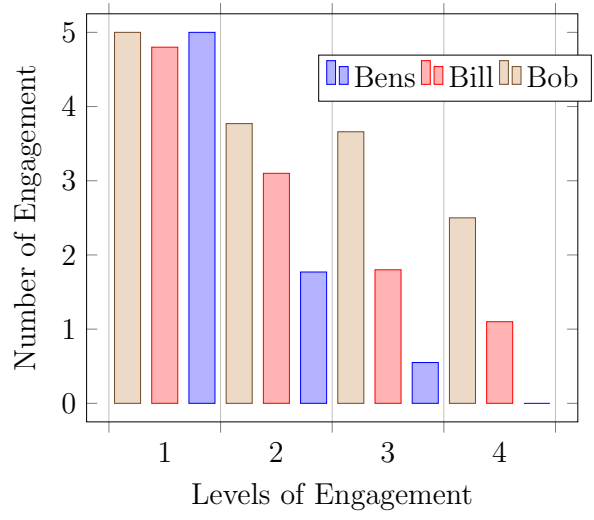

(a) Results without CRI

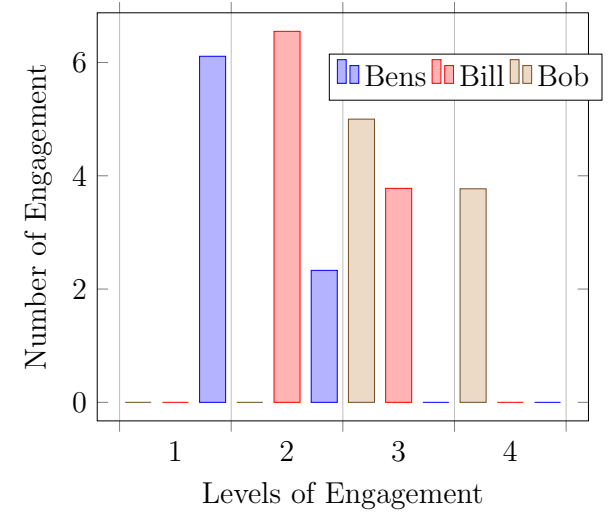

(b) Results with CRI

Figure 9: The histograms show, for each level of interaction, the number of times a patient is engaged by the robot while he is performing a SKT. Figures 9a and 9b point out the differences among the three use-cases in term of number of times and levels of engagement, respectively, without and with a first CRI.

\begin{tabular}{|c|c|c|c|}
\hline Patients & SKT time & n eng & n doing nothing \\
\hline Bens & $22.1 \mathrm{~s}$ & 7.3 & 1.4 \\
\hline Bill & $47.2 \mathrm{~s}$ & 11 & 3.6 \\
\hline Bob & $75.2 \mathrm{~s}$ & 14.4 & 6.2 \\
\hline
\end{tabular}

(a) Results with no CRI

\begin{tabular}{|c|c|c|c|}
\hline Patients & SKT time & n eng & n doing nothing \\
\hline Bens & $31.2 \mathrm{~s}$ & 8.7 & 2.2 \\
\hline Bill & $38.5 \mathrm{~s}$ & 10.3 & 3.3 \\
\hline Bob & $31.8 \mathrm{~s}$ & 8.4 & 2.6 \\
\hline
\end{tabular}

(b) Results with CRI

Table 6: In Table 6a we summarize the experiments results of our three use cases without a first CRI. While, in Table 6b the results with a first loop of interaction with the caregiver are illustrated.

much support from the robot. He only needed to be encouraged and sometimes to get suggestions, if he did not know what to do or if he made a wrong move. Moreover, it was very infrequent that he lost attention. The average number of times he was engaged was 7.3 and the average time to accomplish SKT was 22.1 seconds (Table 6a).

Figure $8 \mathrm{~b}$ shows the evolution in time of Bill playing SKT. In that case, it is possible to notice that Bill needed more assistance to complete the task. The robot engaged him 11 times. Sometimes he waited for the robot to suggest him the correct position of the block on the board and, only in that case, he could successfully accomplish the task.

He took 47.2 seconds to move all the blocks to the correct positions (Table 6a).

Figure 8c shows Bob interaction with the robot while playing SKT. Bob 


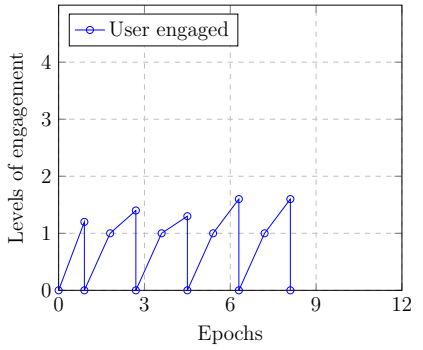

(a) Bens very mild dementia, average time $31 \mathrm{~s}$

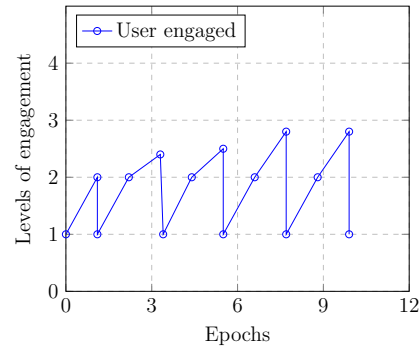

(b) Bill mild dementia, average time $35 \mathrm{~s}$

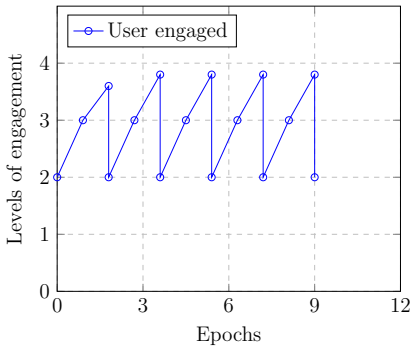

(c) Bob severe dementia, average time $32 \mathrm{~s}$

Figure 10: The plots show, for each patient, the interaction levels employed by the robot in order to support and assist him with a first CRI.

had severe dementia and without the robot assistance, it would be quite impossible for him to perform the moves and succeed. He was engaged 14.4 times by the robot and he took 75.2 seconds to complete the test.

In conclusion, patients with very mild dementia (like Bens) were able to complete the test with very few actions, while patients with severe dementia (like Bob) needed a lot of attempts and assistance for completing it. Moreover, there were significant differences in terms of completion time to achieve the goal by the three patients.

\subsection{Experiments with Caregiver-Robot Interaction}

A second batch of experiments, with the same procedure as before, was performed with a preliminary CRI. The objective here was to evaluate whether a first engagement of the caregiver with the robot could improve the final score performance of the patient and contribute to his overall positive attitude. The results for each patient are shown in the plots of Figure 10. The plots show, for each patient, the interaction levels employed by the robot in order to support and assist him with a first CRI. In this case, the robot firstly interacted with the caregiver, which set up the patient physical and mental impairment on the system, then it engaged the patient with the proper levels of assistance and support as shown in Figure 9b.

As it is shown in Table 6b, the average time to complete SKT was very similar for all the patients. The same kind of results were obtained for the other two measured parameters: number of engagements and number of doing nothing actions. This result suggests that, with the proper engagement, a patient with severe dementia can also complete the task in a reasonable 
time. Obviously in the case of Bob (Figure 10c) most of the work was performed by the robot. However, at the end of the game the patient could have learned the movements and the correct locations for the blocks and could be able to play again next time with less support from the robot.

\subsection{Discussion}

The experiments confirm what we expected. The results obtained over a set of tests for each patient suggest that: if engaged in a proper way, elderly people suffering of MCI and/or AD could improve their score to play SKT, a cognitive test designed for assessing deficits of memory and attention.

For example, Bens took 22 seconds to complete the task with no CRI (Table 6a), while he took 31 seconds with CRI (Table 6b). The results were better without CRI because he had been assisted sometimes too much for his level of impairment (Figure 8a). On the other hand, with a CRI, the robot provided Bens only the engagement levels assigned by the caregiver: it gave encouragement and sometimes suggestions but it never told him the correct solution, and the patient had to guess the proper move by himself. The plots in Figure 8a and Figure 10a show that trend.

Different considerations merit Bill and Bob. In both cases, we notice that although the trend of the curve of the engagement levels along time was higher, see Figure $8 \mathrm{~b}$ and Figure $8 \mathrm{c}$, the selection of lower levels of engagement (Eng_1 for Bill and Eng_1 and Eng_2 for Bob), compared to the expected ones for those patients (Eng_2 and Eng_3 for Bill and Eng_3 and Eng_4 for Bob), increased the time to complete the task. In other words, patients with mild dementia or severe dementia had a very low probability of guessing the proper move if they were only encouraged. They needed their levels of engagement to complete the test and achieve the goal, as shown in Figure $10 \mathrm{~b}$ and Figure $10 \mathrm{c}$.

Other important aspects that deserve a deeper analysis are the patient satisfaction and their aptitude to re-make the test again. For patients like Bill and Bob, in the case of no CRI, the time to complete the task, as reported in Table 6a, could be considerably long, and during the test they could lose attention most of the time and feel frustrated by not succeeding in achieving the goal. For some patients, like Bens, in the case of no CRI, the time to complete the test could be too short due to the excessive support by the robot, and they could get bored because most of the task was performed by the robot. 
With a CRI, the caregiver, can set up the correct level of engagement, and he can prevent those situations, preserving the positive attitude of the patient.

In conclusion, the results of the experiments support both our hypotheses. First, our robot was able to improve and maintain the cognitive attention of the patients with dementia and/or cognitive impairments through its encouragement and support, at different levels, based on administrating SKT. Secondly, thanks to the loop of interaction with the caregiver, the robot's capability of adapting its behavior to the patient's level of impairment helped to maximize the user's task performance in the cognitive test (SKT).

\section{Conclusions}

In this paper we present a symbolic decision system formalized in PDDL that can provide training and evaluation of a patient cognitive level. Our model focuses on engaging the user through speech and gesture that can assist him partially or fully while performing a cognitive exercise.

The simulated experiments show promising results. When the robot interacts with the patients, without any information about their mental and physical impairments, it has a predefined behavior and engages with all of them in the same way. The results show that a standard behavior is not a proper way of interaction because, in some cases, the level of engagement is too low and in some others it is too high. This could affect the patient's performance and his overall positive attitude.

On the contrary, for keeping patients engaged, we decided to personalize the robot behavior through a first interaction with the caregiver that can adequately set it up coherently with the patient impairments, thus making the test neither too easy nor too difficult for the patient.

We expect to evaluate our system in a real scenario, providing a tool to the caregiver for monitoring the progress or regress of the patient and evaluating it from a medical point of view.

\section{Acknowledgements}

We would like to thank Carla Abdelnour and Natalia Tantinya from Fundació ACE for the fruitful discussions and the help in the design of the SKT-based tests. 
This project has received funding from the European Union's Horizon 2020 research and innovation programme under the Marie Skłodowska-Curie grant agreement No 721619 by the Spanish Ministry of Science and Innovation under HuMoUR TIN2017-90086 and by the Spanish State Research Agency through the María de Maeztu Seal of Excellence to IRI (MDM-20160656).

\section{Appendix A. PDDL code for Patient-Robot Interaction}

In this section we include the git repository (https://gitlab.iri.upc . edu/perception/skt_planning_domain) with the code we developed for the problem and the domain definition. The former contains the domain predicates and actions of the actors involved in the interaction. The latter includes the problem instance, initial state description and the goal to be achieved. Please note that the goal and the state description change dynamically after each interaction of the robot with the user, according to our definition of subGoals. 


\section{Appendix B. List of Abbreviations}

HRI ........ Human-Robot Interaction

CRI ........ Caregiver-Robot Interaction

PRI ........ Patient-Robot Interaction

SKT ......... Syndrom Kurztest neuropsychological battery

SAR ........ Socially Assistive Robotics

AD $\ldots . . \ldots \ldots$ Alzheimer's Disease

MCI ......... Mild Cognitive Impairment

PDDL ....... Planning Domain Definition Language

ADL ......... Action Description Language

STRIPS ..... Stanford Research Institute Problem Solver

FSM ........ Finite State Machine

BT $\ldots . . . \ldots$ Behavior Trees

FF $\ldots \ldots \ldots$ Fast-Forward 


\section{References}

[1] World Health Organization, Dementia: a public health priority, Dementia (2012) 112.

[2] R. Mayeux, Y. Stern, Epidemiology of Alzheimer disease, Cold Spring Harbor Perspectives in Medicine 2 (2012).

[3] M. Prince, A. Comas-Herrera, M. Knapp, M. Guerchet, M. Karagiannidou, World Alzheimer Report Improving healthcare for people living with dementia. Coverage, Quality and costs now and in the future, Alzheimer's Disease International (ADI) (2016) 1-140.

[4] National Academy on an Aging Society, Caregiving: Helping the elderly with activity limitations, volume no. 7, Challenges for the 21st Century: Chronic Disabling Conditions, 2000.

[5] J. E. Overall, R. Schaltenbrand, The SKT neuropsychological test battery, Journal of Geriatric Psychiatry Neurology 5 (1992) 220-227.

[6] H. Erzigkeit, SKT: a short cognitive performance test for assessing deficits of memory and attention. User's Manual., International Psychogeriatrics 9 (2001) 115-121.

[7] U. Schramm, G. Berger, R. Müller, T. Kratzsch, J. Peters, L. Frölich, Psychometric properties of Clock Drawing Test and MMSE or Short Performance Test (SKT) in dementia screening in a memory clinic population, International journal of geriatric psychiatry 17 (2002) 254-260.

[8] P. Squarzoni, J. Tamashiro-Duran, F. Luis, S. Duran, L. C. Santos, H. P. Vallada, P. Rossi Menezes, M. Scazufca, G. B. Filho, T. Correa, T. De Ferraz Alves, Relationship Between Regional Brain Volumes and Cognitive Performance in the Healthy Aging: An MRI Study Using Voxel-Based Morphometry, Journal of Alzheimer's Disease 31 (2012) $45-58$.

[9] M. Ibarria, M. Alegret, S. Valero, A. Morera, M. Guitart, P. Canabate, M. Moreno, S. Lara, S. Diego, J. Hernandez, N. Tantinya, M. Vera, I. Hernandez, J. T. Becker, A. Ruiz, M. Boada, L. Tarraga, Beneficial Effects of an Integrated Psychostimulation Program in Patients with 
Alzheimer's Disease, Journal of Alzheimer's disease : JAD 50 (2015) $559-566$.

[10] M. Milders, S. Bell, A. Lorimer, T. MacEwan, A. McBain, Cognitive stimulation by caregivers for people with dementia, Geriatric Nursing 34 (2013) 267-273.

[11] M. Cristina Nascimento Dourado, J. Laks, Psychological Interventions for Neuropsychiatric Disturbances in Mild and Moderate Alzheimer's Disease: Current Evidences and Future Directions, Current Alzheimer Research 13 (2016) 1100-1111.

[12] J. A. Anguera, J. Boccanfuso, J. L. Rintoul, O. Al-Hashimi, F. Faraji, J. Janowich, E. Kong, Y. Larraburo, C. Rolle, E. Johnston, A. Gazzaley, Video game training enhances cognitive control in older adults, Nature 501 (2013) 97-101.

[13] J. Wiemeyer, A. Kliem, Serious games in prevention and rehabilitationa new panacea for elderly people?, European Review of Aging and Physical Activity 9 (2012) 41-50.

[14] S. McCallum, C. Boletsis, Dementia games: A literature review of dementia-related serious games, in: Lecture Notes in Computer Science (including subseries Lecture Notes in Artificial Intelligence and Lecture Notes in Bioinformatics) 2013, volume 8101 LNCS, pp. 15-27.

[15] A. Cooper, The Inmates are Running the Asylum, Macmillan Publishing Co., Inc., Indianapolis, IN, USA, 1999.

[16] M. Montemerlo, J. Pineau, N. Roy, S. Thrun, V. Verma, Experiences with a mobile robotic guide for the elderly, Proceedings of the National Conference on Artificial Intelligence (2002) 587-592.

[17] C. D. Kidd, W. Taggart, S. Turkle, A sociable robot to encourage social interaction among the elderly, Proceedings - IEEE International Conference on Robotics and Automation (2006) 3972-3976.

[18] Y. Matsusaka, H. Fujii, T. Okano, I. Hara, Health exercise demonstration robot TAIZO and effects of using voice command in robothuman collaborative demonstration, in: Proceedings - IEEE International Workshop on Robot and Human Interactive Communication (2009), pp. 472-477. 
[19] T. Hamada, H. Okubo, K. Inoue, J. Maruyama, H. Onari, Y. Kagawa, T. Hashimoto, Robot therapy as for recreation for elderly people with dementia - Game recreation using a pet-type robot -, Proceedings of the 17th IEEE International Symposium on Robot and Human Interactive Communication, RO-MAN (2008) 174-179.

[20] D. McColl, G. Nejat, Meal-Time with a Socially Assistive Robot and Older Adults at a Long-term Care Facility, Journal of Human-Robot Interaction 2 (2013) 152-171.

[21] A. Tapus, C. Țăpuş, M. J. Matarić, The use of socially assistive robots in the design of intelligent cognitive therapies for people with dementia, IEEE International Conference on Rehabilitation Robotics (2009) 924929.

[22] W. A. Bainbridge, J. W. Hart, E. S. Kim, B. Scassellati, The benefits of interactions with physically present robots over video-displayed agents, International Journal of Social Robotics 3 (2011) 41-52.

[23] D. Chisholm, P. Toto, K. Raina, M. Holm, J. Rogers, Evaluating capacity to live independently and safely in the community: Performance Assessment of Self-care Skills, British Journal of Occupational Therapy 77 (2014) 59-63.

[24] A. Marzinotto, M. Colledanchise, C. Smith, P. Ogren, Towards a unified behavior Trees framework for robot control, in: Proceedings - IEEE International Conference on Robotics and Automation (2014), pp. 54205427. 